\title{
Las trayectorias escolares, un análisis por cohorte generacional
}

\section{School trajectories, an analysis by generational cohort}

LEGORRETA-BARRANCOS, Leydi Elena†े, ORTEGA-RODRÍGUEZ, Ana Luisa, GARCÍA-PÉREZ, Sergio Francisco y MAY-SALAZAR, Laura Angélica

Instituto Tecnológico de Campeche, México.

Secretaría de Educación Pública, México.

ID $1^{\mathrm{er}}$ autor: Leydi Elena, Legorreta-Barrancos / ORC ID: 0000-0002-8918-4226, CVU CONACYT ID: 725501

ID $1^{\text {er }}$ Coautor: Ana Luisa, Ortega-Rodríguez, ORC ID: 0000-0002-0134-2008, CVU CONACYT ID: 228237

ID $2^{\text {do }}$ Coautor: Sergio Francisco, García-Pérez / ORC ID: 0000-0002-4538-068X, CVU CONACYT ID: 997074

ID $3^{\text {er }}$ Coautor: Laura Angélica, May-Salazar / ORC ID: 0000-0002-9972-162X, CVU CONACYT ID: 913283

DOI: $10.35429 / J H S .2020 .12 .4 .1 .11$

Recibido Julio 10, 2020; Aceptado Diciembre 30, 2020

\section{Resumen}

El estudio y seguimiento de las trayectorias escolares en las Instituciones de Educación Superior constituyen una fuente de información, que sistematizada es capaz de fortalecer la planeación educativa, en la búsqueda de mejorar la calidad y pertinencia de los servicios educativos, así como contribuir al logro de una educación superior más inclusiva. Se presenta el resultado de un estudio de trayectorias escolares generacionales del área de Ciencias Económico - Administrativas del Tecnológico Nacional de México/Campus Campeche; se utilizó un enfoque cuantitativo, de alcance descriptivo a partir de los registros existentes en el Sistema Integral de Información (SII). Atendiendo la complejidad del objeto de estudio, se caracterizó a los estudiantes en términos de egreso, baja definitiva, deserción, traslado, inscritos, rezago, activos y titulación, entre otros aspectos, permitiendo identificar el comportamiento del desempeño escolar en los programas de estudio adscritos: Ingeniería en Gestión Empresarial e Ingeniería en Administración del ciclo 2010 - 2018.

Educación Superior Tecnológica, Trayectorias escolares, Rendimiento académico

\begin{abstract}
The study and monitoring of school careers in Higher Education Institutions constitute a source of information that, systematized, is capable of strengthening educational planning, seeking to improve the quality and relevance of educational services, as well as contributing to the achievement of a more inclusive higher education. The result of a study of generational school trajectories in the area of Economic and Administrative Sciences of the National Technological Institute of Mexico / Campeche Campus is presented; A quantitative, descriptive scope approach was used from the existing records in the Comprehensive Information System (SII). Taking into account the complexity of the object of study, the students were characterized in terms of graduation, definitive dropout, desertion, transfer, enrolled, lag, assets and degree, among other aspects, allowing the identification of the behavior of school performance in the attached study programs: Engineering in Business Management and Engineering in Administration of the 2010-2018 cycle.
\end{abstract}

Higher Technological Education, School Pathways, Academic Performance

Citación: LEGORRETA-BARRANCOS, Leydi Elena, ORTEGA-RODRÍGUEZ, Ana Luisa, GARCÍA-PÉREZ, Sergio Francisco y MAY-SALAZAR, Laura Angélica. Las trayectorias escolares, un análisis por cohorte generacional. Revista de Educación Superior. 2020. 4-12:1-11.

$\uparrow$ Investigador contribuyendo como primer autor. 


\section{Introducción}

Ante el compromiso de transformar la escuela de México, el Subsistema de Educación Superior (SES), indudablemente representa una oportunidad para la generación de comunidades de aprendizaje de alto desempeño, la innovación y el desarrollo tecnológico; capaces de modificar las estructuras sociales a través de las distintas áreas del conocimiento y responder así, a los desafíos planteados por el Tecnológico Nacional de México (TecNM) como son: cobertura, calidad y pertinencia para el desarrollo regional y los avances del nuevo Modelo Educativo del siglo XXI.

Bajo un contexto global de cambios vertiginosos, y la insuficiente eficiencia de egreso se requieren mejores mecanismos para evaluar los resultados del proceso de enseñanza y los comportamientos de los diferentes grupos de estudiantes, siendo necesario profundizar en la búsqueda de nuevos factores que intervienen en la ruta que trazan y las estrategias que desarrollan en su tránsito por la vida escolar tecnológica.

Diversos estudios han demostrado que la Eficiencia Terminal (ET), sigue siendo un reto a superar, al evaluar la importancia de aquellos elementos que caracterizan a los estudiantes; sin embargo, para la política actual resulta insuficiente al determinar el TecNM en su diagnóstico de continuidad, la tarea de estudiar las Trayectorias Escolares de manera permanente, como un aspecto clave que contribuye al diseño de estrategias, acciones y políticas institucionales que favorezcan el desempeño institucional (TecNM, 2019); por tal motivo, se describe, cuantitativamente, la continuidad y/o discontinuidad en el ritmo temporal en los estudios correspondientes a las licenciaturas de Ingeniería en Gestión Empresarial (IGE) e Ingeniería en Administración (IA) por generación o cohorte del 2010 al 2018.

\section{Educación Superior Tecnológica}

En México la Educación Superior Tecnológica, ha desafiado diversas dificultades relacionadas con la calidad educativa, mismas que se expresan en una serie de indicadores como son: cobertura, infraestructura, planta académica, programa curricular, calidad de la formación profesional, calidad del profesorado, producción científica y eficiencia terminal, entre otros (Rugarcía, 1994; ANUIES, 2009 en Garcia y Barron, 2011).

La transformación de su quehacer académico y compromiso social, se han sustentado en la reforma de los modelos educativos, la reformulación de la oferta educativa $\mathrm{y}$ en cambios administrativos $\mathrm{y}$ organizacionales; en respuesta a la emergencia económica, acelerado cambio tecnológico, nueva organización social de la producción, nuevas demandas y perfiles profesionales requeridos; siendo la última reforma el 23 de julio de 2014 (DOF. 2014, 07 23) que da origen al TecNM.

Desde entonces, órgano desconcentrado de la Secretaría de Educación Pública (SEP), considerado como la institución más grande de la educación superior del país y en Latinoamérica, actualmente cuenta con 254 instituciones de educación superior tecnológica: 132 Federales (126 Institutos Tecnológicos, cuatro Centros Regionales de Optimización y Desarrollo de Equipo y dos Centros de Investigación), así como 122 Institutos Tecnológicos Descentralizados, distribuidos en las 32 entidades del país; representando el 12.9 $\%$ de la matrícula de Educación Superior de México (PTA, 2019). 
Atendiendo al Plan Nacional de Desarrollo (PND) 2019 - 2024, la SEP asume la tarea de transformar la Educación Superior en cuanto a condiciones de desigualdad y calidad de la enseñanza, con el propósito de generar mejores beneficios a la sociedad mexicana; para emprender este mandato, el TecNM retoma su Programa Institucional de Innovación y Desarrollo (PIID, 2014) 20132018, que fue parte del cierre de la administración anterior y punto de partida para dar continuidad a las funciones encomendadas, tal y como se señala en el Programa Anual 2019, poniendo en claro su propósito, "formar profesionales e investigadores con habilidades y competencias suficientes para generar y aplicar conocimiento, que permita solucionar problemas económicos, sociales y ambientales"; así como los 23 indicadores a los que da seguimiento para atender los problemas identificados y clasificados en cuatro rubros: Infraestructura, Personal Académico, Docencia e Investigación y Gobernanza.

Es importante mencionar que el diagnóstico que se presenta en el PTA 2019 permitió determinar diversas áreas de oportunidad, los ejes estratégicos, acciones e indicadores para su atención, señalando únicamente aquel que justifica el tema que nos ocupa (Ver Tabla 1).

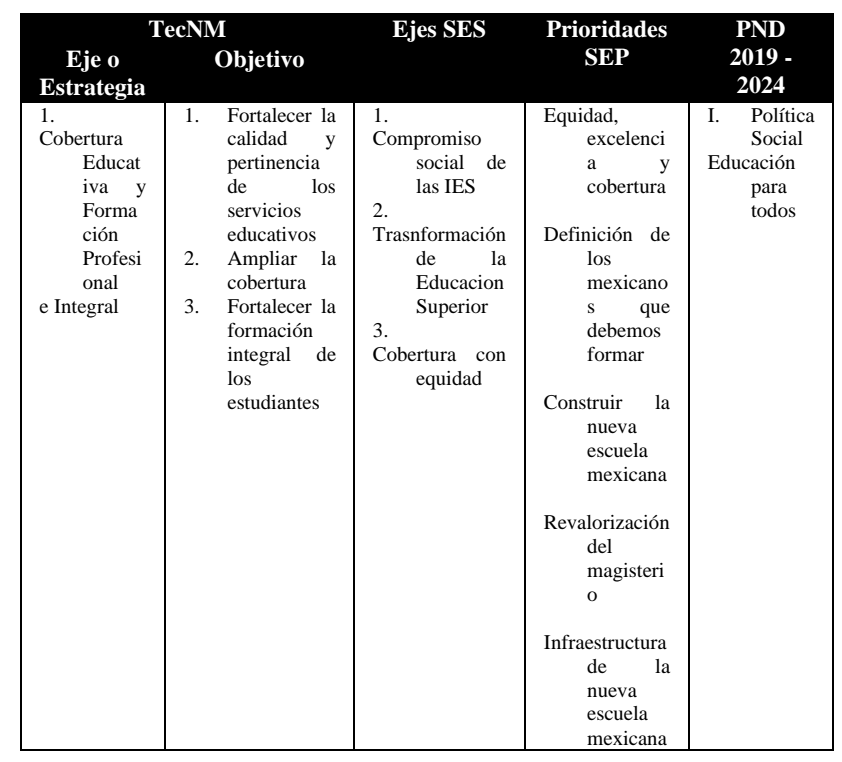

Tabla 1 Alineación con el PND 2019-2024 y Prioridades de la SEP y SES (PTA, 2019), p. 17
Como puede observarse, el TecNM considera entre sus objetivos Amplear la cobertura; y para ello, presenta su línea de acción 2.2 Garantizar la permanencia y egreso de estudiantes, a través del seguimiento permanente de su Trayectoria Escolar y de los programas de Becas y Tutorías; lo que justifica el estudio que se presenta.

Es importante mencionar que los objetivos considerados para este eje estratégico, están relacionados con los resultado obtenidos en los indicadores que presenta en el 2018 (Continuidad PIID 2013 - 2018) como se observa en la siguiente tabla.

\begin{tabular}{|c|c|c|c|}
\hline Indicador & $\begin{array}{c}\text { Meta } \\
2018 \\
\text { (PID) }\end{array}$ & $\begin{array}{c}\text { Alcanzado } \\
\text { en } 2018\end{array}$ & $\begin{array}{c}\text { Programado } \\
2019\end{array}$ \\
\hline $\begin{array}{l}2.1 \\
\text { Matrícula } \\
\text { del nivel } \\
\text { licenciatu } \\
\text { ra }\end{array}$ & 602,084 & 602,535 & 624,500 \\
\hline $\begin{array}{l}2.5 \\
\text { Eficiencia } \\
\text { terminal }\end{array}$ & $\begin{array}{r}70.00 \\
\%\end{array}$ & $56.24 \%$ & $70 \%$ \\
\hline
\end{tabular}

Tabla 2 Indicador de continuidad al PIID 2013 - 2018 Fuente Elaboración propia compilado del PIID 2013 2018, p. 19

Se denota un logro en el incremento de la matrícula por encima de la meta establecida; sin embargo, la ET como indicador de desempeño, al parecer resulta insuficiente para llegar a la meta planteada; por lo cual, se considera oportuno identificar los comportamientos de riesgo reales del estudiante durante su estancia escolar; pues se desconocen los momentos en los cuáles el de abandono se incrementa en cada plan y programa de estudio; por lo que surgen los siguientes cuestionamientos ¿cuáles son los indicadores que se consideran para estudiar la trayectoria escolar? ¿cuáles son los semestres que resultan más difíciles para el estudiante?¿se tiene identificado los temas de aquellas asignaturas que mantienen una constante de reprobación?; ¿de qué manera se están atendiendo a los estudiantes en riesgo?; con base a estos, es conveniente incorporar otros criterios que amplien el conocimiento y coadyuve al fortalecimiento de la calidad y pertinencia de los servicios educativos. 


\section{Acerca de las trayectorias escolares}

Es común encontrar estudios sobre deserción o rezago, pero poco se habla de aprovechamiento o rendimiento escolar. Recién se ha comprendido que para entender la eficiencia terminal tiene que entenderse el conjunto de los fenómenos del proceso escolar; y en este sentido, los estudios sobre trayectoria escolar cobran relevancia a partir de los ochentas ante el complejo conjunto de problemas que afectan la regularidad del comportamiento académico.

Según Barranco y Santacruz (1995 citado en García O. y Barrón C., 2011) los estudios han descrito los fenómenos del comportamiento académico de un individuo, incluyendo el desempeño escolar, la aprobación, la reprobación, el promedio logrado, entre otros, a lo largo de los ciclos escolares especificados en una cohorte; de manera adicional Cantero (2003 citado en Tovar, Gutiérrez y Sustay, 2017), señala la importancia de explicar los factores que provocan dicho comportamiento y su intervención en el éxito escolar; estudios como el de McKenzie y Schweitzar (2001 en Tovar, Gutiérrez y Sustay, 2017), destacan cuatro factores relacionados con el desempeño académico de los alumnos universitarios: económicos, psicosociales, apreciación cognitiva y demográficos; Cuevas (2001, citado en Sánchez, C, 2019) considera el análisis de factores cualitativos que describen los aspectos psicológico y sociológico, y los cuantitativos que proporcionan datos precisos sobre los resultados académicos tanto de los estudiantes como de la misma institución, con la finalidad de diseñar acciones encaminadas a lograr un mejor trayecto del alumno en la institución educativa.

Chain en 1997 (citado en Casillas, M., Chain, R., \& Jácome, N., 2015) demostró en su estudio, que la trayectoria escolar de los estudiantes en el marco de la estructura formal del plan de estudios: "[...] está compuesta de diferentes comportamientos académicos relativos al rendimiento escolar, aprobación, reprobación, repetición, rezago y deserción; variables, todas ellas, que determinan diferentes trayectorias académicas" (ANUIES, 2005; 2007, pp. 26-27); desde entonces este autor es roconocido como uno de los precursores del campo de las trayectorias escolares en México.
En este sentido, hablar de las trayectorias escolares, es hablar de la historia académica de los estudiantes desde su ingreso hasta la culminación de sus estudios profesionales (DEGEST, 2013) y para estudiar la trayectoria escolar, Bautista y Roldán (1996, citado en Fernández, J., Peña, A. y Vera, F., 2006) consideran que los criterios que se deben analizar son: avance escolar, rendimiento escolar, eficiencia terminal, egreso, rezago y abandono escolar.

Desde el Modelo de dimensiones de Chain (1997, en Fernández, J., Peña, A. y Vera, F. 2006), los criterios se categorizan en tres dimensiones y son los que hasta la fecha han sido adoptados por la SEP y distintas Universidades:

Dimensión Tiempo, se refiere a la continuidad y/o discontinuidad en el ritmo de estudio del alumno en correspondencia con su generación (regular/irregular). El periodo de la transición de los programas educativos del sistema de los tecnológicos, comprende 9 semestres y por su flexibilidad, puede alargarse el tiempo de instancia del estudiante hasta doce semestres (TecNM, 2015).

- Dimensión Eficiencia escolar, a la forma en que los estudiantes transitan por las asignaturas, y que categoríza en:

- Ordinario: estudiantes que promueven todas las asignaturas en el tiempo determinado por el plan de estudios.

- No ordinario: estudiantes que promueven asignaturas en modalidades distintas al examen ordinario.

En este caso el TecNM (2015) señala en sus lineamientos que para acreditar una asignatura el estudiante, tiene derecho a la evaluación de primera oportunidad y evaluación de segunda oportunidad en los cursos ordinario, repetición y especial. 
Dimensión Rendimiento escolar, al promedio simple de calificación obtenido por el alumno en las asignaturas en las cuales ha presentado examen, independientemente del tipo de éste.

En este caso se considera el promedio de calificaciones por generación.

Camarena Rosa y otros (2004 citado en Zandomeni y Canale, 2010), consideran que el estudio de las trayectorias escolares en el nivel de educación superior tecnológica, tiene el atributo de hacer visibles las condiciones académicas de los estudiantes en tres momentos: el ingreso, el tránsito por el instituto y el egreso.

El ingreso: se refiere a los datos que arrojan los exámenes de admisión, permite conocer mejor las características de los estudiantes de nuevo ingreso y de sus demandas académicas.

- El tránsito académico: tránsito del estudiante por la institución académica, mismo que permite llevar registros del comportamiento de su desempeño a través de los semestres.

El egreso: arroja datos acerca de las condiciones académicas en que la población de estudiantes concluye, con la obtención del certificado; lo que se conoce como Eficiencia de Egreso (EE).

Durante la estancia escolar del estudiante del TecNM (2015), se presentan opciones académicas vigentes, que influyen en el logro de las metas institucionales, que al mismo tiempo implica una oportunidad o una amenaza dependiendo de las condiciones de los servicios educativos de apoyo que brindan las instituciones, así como la pertinencia de sus planes y programas de estudio, por lo que se considera necesario conceptualizar algunos aspectos para una mejor comprensión.

Estudiante de nuevo ingreso: en educación superior, se designa así al estudiante que se matricula o inscribe por primera vez en un programa educativo.
Estudiante activo en el sistema sin inscripción: estudiante cuyos registros obran en el SII y se considera que aún forma parte de la matrícula institucional, pero que se desconoce el por qué no se encuentran inscritos, ni dados de baja definitiva, ni temporal como se establece en la política 5.4.6.8 del Manual de lineamientos Académico Administrativo del TecNM versión 1.0 (2015) para la acreditación de asignaturas "cuando haya agotado los doce periodos escolares semestrales permitidos como máximo para concluir su plan de estudios" p. 28.

Eficiencia de Egreso: $(\mathrm{EE})=($ Total de egresados de la institución en el ciclo escolar $\mathrm{n} /$ total de alumnos de nuevo ingreso 5 años antes)*100

Estudiante activo en el sistema con rezago: estudiante que presenta una condición de atraso en la inscripción a las asignaturas que, según la secuencia del plan de estudios, debería cursar en un momento determinado, de acuerdo a la cohorte generacional a la cual pertenecen, $o$ al egreso de la misma (ANUIES, 2001; De los Santos, 2004 citado en García y Barrón, 2011).

En el TecNM se considera cuando el estudiante no acredita una asignatura por primera vez y se le ofrece el curso de repetición que debe cursarse de manera obligatoria en el período posterior al que no acreditó la asignatura, siempre y cuando ésta se ofrezca; en este momento de tránsito es pertinente analizar por la instancia correspondiente la cantidad de estudiantes que requieren recursarla o bien la dimensión de su impacto en los créditos para concluir en tiempo su programa de estudio (TecNM, 2015 p. 18).

Baja definitiva: el estudiante causa baja definitiva por no acreditar el curso especial, tendrá la oportunidad de un solo reingreso al mismo Instituto a un plan de estudios diferente - de acuerdo con la recomendación del Comité Académico - siempre y cuando el Instituto cuente con las condiciones, recursos y de acuerdo con la capacidad del mismo, cumpliendo el proceso de admisión (TecNM, 2015 p. 27). 
Traslado o movilidad estudiantil: es el acto legal que reconoce que un estudiante adscrito en un instituto tecnológico cambia de adscripción a otro adscrito al TecNM, conservando los derechos y obligaciones que le proporciona ser estudiante de los institutos (TecNM, 2015 p. 29).

Salida lateral: estrategia educativa institucional, para aquellos estudiantes que debido a circunstancias, que no estén a su alcance le impidan concluir el plan de estudios a nivel superior $y$ que le permite el reconocimiento de un conjunto de competencias genéricas y específicas que desarrolló durante su formación, para su incorporación inmediata al sector productivo y de servicios, cumpliendo con la aprobación de un mínimo de $60 \%$ de los créditos de su plan de estudios más una asignatura de especialidad (TecNM, 2015 p. 29).

Cohorte: grupo de personas que comparten características similares entre sí o que experimentan una misma situación durante determinado lapso. Por ejemplo: un grupo de alumnos que terminan un ciclo educativo específico (Salazar, et al. 2019).

\section{Rendimiento académico}

Se ha demostrado que el rendimiento académico en su carácter multifactorial, está condicionado por la interrelación de un conjunto de factores (Vásquez, 2012; Cerquera, 2014 citados en Velasco, L., 2016); siendo común para la escuela medir con indicadores cuantitativos expresados en una calificación escolar, lo que testifica que una persona ha aprendido, como resultado de un proceso de instrucción o formación (Vergara, 2011 citado en Fernández, 2018).

Estas valoraciones del rendimiento académico que realiza la escuela, para Martínez-Otero (2009), se estiman generalmente la adquisición de conocimientos y competencias del alumnado, y los avances - $\mathrm{O}$ retrocesos- en la ejecución del currículo, materias ganadas o perdidas, la deserción y el grado de éxito académico

\section{Metodología}

El estudio que se presenta es un primer acercamiento a las trayectorias escolares de los estudiantes adscritos en los al área de Ciencias Económicas - Administrativas del TecNM/ Campus Campeche.

La investigación se realizó con enfoque cuantitativo de tipo exploratoria de alcance descriptivo, pues "su objetivo es examinar un tema o problema de investigación poco estudiado o que no ha sido abordado antes" (Hernández y otros, 1994:59); este estudio corresponde al período 2010 al 2018, identificando el comportamiento de los indicadores de ingreso, egreso, bajas, deserción, traslados, inscritos, rezago y activos; permitiendo determinar las áreas potenciales de mejora.

\section{Resultados}

El ingreso a las licenciaturas adscritas al área, se realiza por ciclo escolar anual, y en los cinco cohortes generacionales que se estudiaron puede notarse cierta constancia en el tamaño de los grupos de IGE, los cuales fluctuaron de 34 a 40 estudiantes; respecto a IA que van de 10 a 79 , siendo importante señalar que se recibieron por única vez nuevos ingresos durante los semestres de enero-junio de 2012 y 2013 , sin que ello represente un impacto significativo en el incremento de la matrícula (Ver tabla 3).

\begin{tabular}{|l|r|r|}
\hline Cohorte Generacional & \multicolumn{2}{c|}{ Ingreso } \\
& IGE & IA \\
\hline $2010-2014$ & 34 & 59 \\
\hline $2011-2015$ & 40 & 55 \\
\hline $2012-2016$ & 0 & 10 \\
\hline $2012-2016$ & 40 & 61 \\
\hline $2013-2017$ & 0 & 13 \\
\hline $2013-2017$ & 35 & 79 \\
\hline $2014-2018$ & 38 & 73 \\
\hline Total & $\mathbf{1 8 7}$ & $\mathbf{3 5 0}$ \\
\hline
\end{tabular}

Tabla 3 Estudiantes al área de Ciencias EconómicoAdministrativas por cohorte generacional Fuente Elaboración propia, 2020 
Como puede observarse, el total de estudiantes es mucho mayor en IA (350) que en IGE (187), lo que hace suponer que: existe mayor demanda de la carrera, la promoción y difusión de carreras no se realizan con el mismo énfasis, o pudiera ser consecuencia a la falta de un adecuado estudio de factibilidad para la apertura de nuevas carreras y diseño de especialidades, entre otras.

El análisis por cohorte generacional, considera la relación entre el ingreso y los diferentes indicadores que estudian la trayectoria escolar; siendo conveniente mencionar que cada cohorte tiene un alcance de nueve semestres, que es el tiempo que establecen los programas de estudio, cuya flexibilidad curricular se expande hasta doce semestres para concluir 260 créditos.

\section{Del comportamiento de los estudiantes de IGE}

Los registros del comportamiento de los estudiantes de IGE (Ver tabla 4), determinaron que de los 187 estudiantes inscritos, el $20 \%$ causaron baja definitiva, $3.2 \%$ solicitó traslado, $27.8 \%$ desertaron, $9.6 \%$ entraron en rezago y $4.3 \%$ se mantuvieron activos en el sistema sin inscripción, entre los que causaron baja definitiva y desertaron se estimó el $47.8 \%$, lo que significa que cerca de la mitad de los inscritos de este universo se perdieron durante su tránsito escolar.

Entre los rezagados o egresados de 10 semestres en adelante y activos en el sistema sin inscripción que representan el $13.9 \%$, confirma que a pesar de tener la oportunidad de concluir sus estudios en el máximo de los doce semestres permitidos, han puesto en riesgo la posibilidad de aspirar a mejorar su calidad de vida, obtener mejores oportunidades de inserción al mercado laboral y que también impacta en el indicador Eficiencia de Egreso (EE). Cabe mencionar que los estudiantes que se encuentran en estas condiciones aún son susceptibles a ser rescatados y puedan concluir el programa de estudios conforme al Lineamiento Académico - Administrativos Acreditación de Asignaturas (versión 1.0); el porcentaje de traslados, no se considera significativo, debido a que representan menos del $2 \%$ en todas las generaciones, haciendo notar que de las cinco cohortes, solo en tres se presentaron.
Respecto al incremento de bajas definitivas en la generación 2011 - 2015 se presentaron mayores porcentajes, lo que pudiera ser consecuencia de que durante este período se gestionaron jubilaciones de docentes expertos y las asignaturas fueron asignadas sin considerar los perfiles del profesorado; sin embargo, al último cohorte el resultado se incrementa nuevamente, lo que denota que existen motivos recurrentes que se desconocen.

La deserción durante las primeras cuatro generaciones fue hasta de un $37.1 \%$; sin embargo, en la última generación se observa una disminución considerable. Aparentemente es el reflejo del proceso de formación de los profesores en las asignaturas de especialidad y del conocimiento de las asignaturas que integran el programa de estudio, porque al ser una ingeniería, los estudiantes cursan asignaturas de ciencias exactas durante los dos primeros años y esto pudo impactar respecto a sus expectativas.

Sin embargo, al considerar estas tres últimas variables de manera conjunta, puede decirse que es preocupante que, durante este tiempo, el porcentaje de estudiantes que se perdieron durante su instancia se incremente impactando en un promedio de $50.8 \%$ del universo; debiendo ser analizado desde el seno de la academia para articular el Programa Institucional de tutorías y determinar estrategias efectivas que coadyuven a disminuir dicho porcentaje.

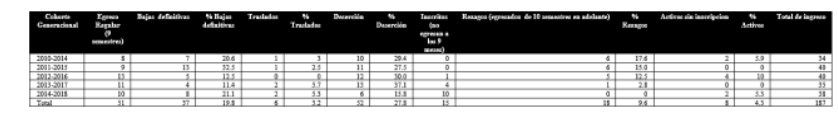

Tabla 4 Comportamiento de Estudiantes de IGE por cohorte generacional

Fuente: Elaboración propia, 2020

Así mismo, se aprecian índices muy bajos de egreso regular (ver tabla 5) y de egreso irregular identificados como estudiantes en rezago, por egresar en un tiempo entre 10 semestres en adelante, ya que el máximo obtenido de egreso regular fue de $32.5 \%$ se encuentra por debajo de la media que corresponde a la generación 2012 - 2016; lo que representa un foco de alarma de la eficiencia del proceso educativo comúnmente conocido como Eficiencia de Egreso (EE) y que decrece en las dos últimas generaciones hasta en un $26.3 \%$. 
En cuanto al rendimiento escolar, se toman los promedios de egreso generacionales, independientemente del tiempo que les tomo lograr la totalidad de los créditos del plan de estudios, colocándose en un rango de desempeño entre 85.39 y 88.43 lo que significa que los estudiantes logran de manera notable sus aprendizajes; sin que esto signifique que no se tenga la necesidad de estrategias que ayuden a transitar hacia un rango de sobresaliente.

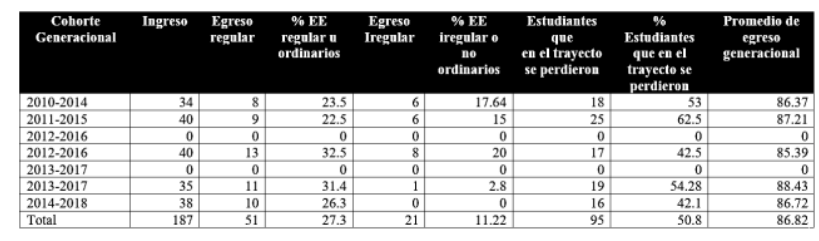

Tabla 5 Eficiencia de Egreso y Eficiencia Escolar Generacional de IGE

Fuente: Elaboración propia, 2020

Lo anterior conlleva a reflexionar acerca del acompañamiento que recibe el estudiante durante su estancia y a revisar de qué medida se atienden sus expectativas; pues pudiera ser que uno de los motivos que incide en prolongar su instancia se deba a que no logren cubrir los créditos necesarios para llevar en tiempo su residencia profesional o por la falta de banco de proyectos para atender la demanda del mismo, la falta de talleres en el área para realizar prácticas innovadoras, entre otras.

\section{Del comportamiento de los estudiantes de IA}

En la Licenciatura de IA se encontraron 350 estudiantes inscritos (Ver tabla 6), de los cuales el $14 \%$ causaron baja definitiva, $2.3 \%$ solicitó traslado, $16 \%$ desertaron, $20 \%$ entraron en rezago y $4 \%$ se mantuvieron activos en el sistema sin inscripción; entre los que causaron baja definitiva y desertaron se halló el $30 \%$, lo que significa que cerca de una tercera parte de la matrícula de estudiantes inscritos se perdió durante su tránsito escolar.

Las bajas definitivas no rebasan el $10 \%$ en ninguno de los cohortes generacionales, lo cual es satisfactorio respecto al universo de inscritos, denotando que de las dos licenciaturas, es en ésta en donde aparentemente se otorga mayor atención en los servicios de apoyo y asistencia a la educación o que existe mayor demanda por la comunidad de estudiantes.
El porcentaje de traslados, no se considera significativo, debido a que fue disminuyendo en cada generación, siendo en el 2014 -2018 del $1.2 \%$, y abría que consultar los expedientes de los estudiantes para conocer los motivos de las solicitudes. La deserción fue hasta de un $16 \%$ de los inscritos, lo cual no representa un foco de alarma, debido a que algunos estudiantes toman la carrera como una segunda opción al no ser aceptados en aquella de su interés vocacional.

El $23.7 \%$ que representan a los rezagados y activos en el sistema sin inscripción, que también se encuentran en riesgo y que pueden recuperar la oportunidad de concluir sus estudios considerando la flexibilidad curricular antes señalada, evitando la frustración de no obtener un certificado escolar y que también impacta en el indicador EE.

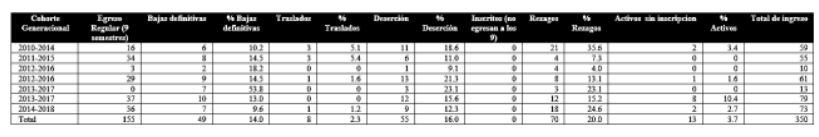

Tabla 6 Comportamiento de Estudiantes de IA por cohorte generacional

Fuente Elaboración propia, 2020

Considerando el grueso de todas las generaciones de esta ingeniería, se observa que el $44.28 \%$ de los estudiantes inscritos, lograron concluir su carrera en nueve semestres; sin embargo, aún quedan brechas en los servicios de apoyo que se les brinda a través de los distintos programas institucionales, y mejorar las estrategias de comunicación para conocer y atender con mayor eficiencia sus necesidades.

En lo que concierte a la eficiencia del proceso educativo o $\mathrm{EE}$, se observa que en agosto - diciembre se incrementa el número de estudiantes inscritos; sin embargo, el egreso regular oscila de $3 \%$ hasta un $37 \%$ (ver tabla 7 ), lo que es preocupante debido a que esta carrera es derivada de la Licenciatura en Administración de Empresas, la cual fue impartida desde hace muchos años y en gran parte por el mismo profesorado. 
Los promedios de egreso generacionales, independientemente del tiempo que les tomo lograr la totalidad de los créditos del plan de estudios, alcanzan un rango de desempeño entre 82.08 y 87.39 lo que significa que los estudiantes de esta ingeniería también logran de manera notable sus aprendizajes; sin que esto signifique que no se tenga la necesidad de estrategias que ayuden a transitar hacia un rango de sobresaliente.

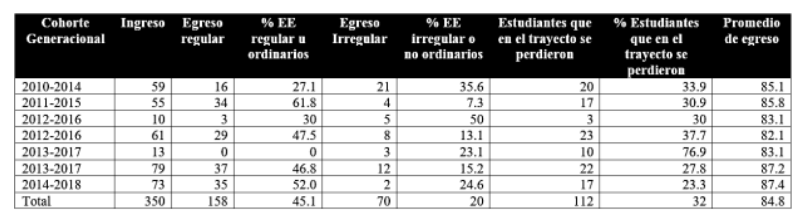

Tabla 7 Eficiencia de Egreso y Eficiencia Escolar Generacional de IA

Fuente Elaboración propia, 2020

Por otra parte, es importante clarificar que en el modelo por competencias para acreditar una asignatura, el estudiante tiene derecho a la evaluación de primera oportunidad y evaluación de segunda oportunidad en los cursos ordinario, de repetición y especial; que sólo existen dos opciones de desempeño en la evaluación de competencias, considerada en los Lineamiento para la Evaluación y Acreditación de Asignaturas Versión 1.0 (TecNM, 2015): Competencia alcanzada o Competencia no alcanzada.

La opción de desempeño de competencia alcanzada está integrada por cuatro niveles de desempeño: excelente, notable, bueno y suficiente; mientras que la opción de desempeño de competencia no alcanzada solo tiene el nivel de desempeño insuficiente.

\section{Agradecimiento}

Gracias al apoyo constante del TecNM/Campus Campeche y en especial al Coordinador de Apoyo a la Titulación de Carrera que colaboró en la obtención de los datos.

\section{Conclusiones}

La Educación Superior Tecnológica, ha dado sobradas pruebas de su viabilidad a lo largo de los años, de su capacidad para transformar, propiciar el cambio y el progreso de la sociedad.
Este tipo de estudios describe el avance y las posibles problemáticas a las que se van enfrentando los estudiantes en los programas educativos, permite planificar e implementar las acciones adecuadas tanto de prevención como de corrección de estas, coadyuvando en las estrategias institucionales y a las exigencias nacionales e internacionales que demanda la calidad educativa; es importante señalar que en toda IES, el buen uso de los datos, limita sistemáticamente la articulación de los procedimientos que impactan en las trayectorias escolares; por lo que se recomienda lo siguiente:

Analizar las estrategias de promoción y difusión de carreras, a fin de identificar aquellas por las que se da la captación de estudiantes de nuevo ingreso, buscando mejorar su planificación, para que, al optar por estas licenciaturas, sean la primera opción en la vocación del estudiante.

Considerar los resultados del perfil de ingreso; es decir, el resultado diagnóstico del Exani-II-Admisión, para generar acciones y prácticas que permitan fortalecer la calidad y pertinencia de los servicios de apoyo a los estudiantes; desarrollar nuevas especialidades, fortalecer la vinculación con las instituciones de educación media superior, y desarrollar planes y programa de estudio pertinentes.

Por otra parte, en necesario diseñar y utilizar herramientas que permitan identificar el riesgo de abandono, pues se desconoce de manera temprana los momentos de riesgo de los estudiantes, desde que entran en rezago y/o se convierten en estudiantes activos en el sistema sin inscripción; y a partir de ello, generar una acción preventiva que disminuya los riesgos de convertirse en bajas y desertores. Emprender el análisis permanente de los diversos factores que caracterizan a las nuevas generaciones, basados en evidencias que denoten la realidad institucional y esto permita tomar decisiones con una visión institucional compartida, haciendo uso de las herramientas y programas presupuestales con las que ya cuenta el sistema, como lo es el Programa Institucional de Tutorías, siendo necesario que el Departamento de Desarrollo Académico establezca un programa de asesorías académicas para abatir el rezago educativo, de acuerdo a los perfiles de los tutores (Legorreta, Ortega, Súarez y García, 2019). 
Fortalecer la vinculación con las instancias y asociaciones para incrementar las becas de estudiantes y generar bancos de proyectos de residencia para ambas ingenierías desde sus respectivas academias.

Identificar las asignaturas con altos índices de reprobación con el propósito de generar comunidades de aprendizaje en las academias, orientadas a fortalecer los programas de formación y actualización del profesorado.

Así, el procedimiento de tutorías, los procesos de planeación, revisión curricular, evaluación, la oferta académica semestral y la prestación de servicios institucionales, deberán atender de manera diferenciada a los estudiantes según sus necesidades, problemas y potencialidades, desmantelando el sistema dominante de creencias y las representaciones sociales que estigmatizan y van desgranando al estudiante.

Si bien es cierto, que para el TecNM el estudio permanente de las trayectorias escolares se han convertido en una necesidad, es importante contar con la creación de un Sistema para el monitoreo de las mismas, que permita calcular los indicadores de análisis y su trasversalidad, y estudiar el aspecto cualitativo, pues no se trata de mirar sólo a un sujeto ni de centrar la atención sólo en la organización escolar, el trabajo de las trayectorias escolares acontece en la relación sistémica que se da entre ambos y sus expectativas.

\section{Referencias}

Asociación Nacional de Universidades e Instituciones de Educación Superior (2001). Deserción, rezago y eficiencia Terminal en las IES. Propuesta metodológica para su estudio. México: Autor.

Bautista, R. (1996). Importancia de los estudios en trayectoria escolar en la educación superio, Planeación y evaluación educativa, vol.10: 2429. México: ANUIES.
Canale, S., Moretti, M., Pacífico, A., y Pagura, M. (2008). Autoevaluación de las carreras de grado de la Facultad de Ciencias Económicas: primeros lineamiento, Revista Ciencias Económicas de la Facultad de Ciencias Económicas, UNL, Volumen 1. https://bibliotecavirtual.unl.edu.ar/publicacione s/index.php/CE/issue/view/128

Cantero, B. (2003). Análisisde los factores que intervienen en la trayectoria escolar del Alumno. Seminarios de diagnóstico locales. Recuperado en abril 01, 2020 de http://www.consejouniversitario.unam.mx:1000 3/archivoCECU/ponsemloc/ponencias/1399.ht $\mathrm{m}$

Casillas, M., Chain, R., \& Jácome, N. (2015). Trayectorias Escolares y Niveles de riesgo en los estudiantes de la Universidad Autónoma de Yucatán. Trayectorias escolares en educación superior. Propuesta metodológica y experiencias en México.

Chaín, R. y Ramírez M. (1997). Trayectoria escolar: la eficiencia terminal en la Universidad Veracruzana Revista de la Educación Superior, núm. 26, 2: 79-97. México: ANUIES.

Dirección General de Educación Superior Tecnológica (2013). Manual del Tutor del SNIT. DEGEST. México.

DOF (2014, 07 23). Decreto que crea al Tecnológico Nacional de México. Diario Oficial de la Federación. Tomo DCCXXX, 21, pp. 47-55. Recuperado en abril 2, 2020 de http://dof.gob.mx/nota_to_pdf.php?fecha=22/0 7/2014\&edicion=MAT

DOF (2019, 07 12). Plan Nacional de Desarrollo. Recuperado en abril 11, 2020 de https://www.dof.gob.mx/nota_detalle.php?codi go $=5565599 \&$ fecha $=12 / 07 / 2019$

Fernández, J., Peña, A. y Vera, F. (206). Los estudios de trayectoria escolar. Su aplicación en la educación media superior. Revista Graffylia , 6, año 3, 24-29. Recuperado en abril 22 , 2020 de https://filosofia.buap.mx/sites/default/files/Graf fylia/6/24.pdf 
Fernández, S. (2018). Rendimiento Académico en Educación Superior: Desafios para el Docente y Compromiso del Estudiante. Revista Científica de la UCSA, Vol. 5 No 3, Diciembre, 5-63 Recuperado en abril 19, 2020 de http://scielo.iics.una.py/pdf/ucsa/v5n3/24098752-ucsa-5-03-55.pdf

García Robelo, Octaviano, \& Barrón Tirado, Concepción. (2011). Un estudio sobre la trayectoria escolar de los estudiantes de doctorado en Pedagogía. Perfiles educativos, 33 (131), 94-113. Recuperado en abril 21, 2020 de

http://www.scielo.org.mx/scielo.php?script=sci _arttext\&pid=S0185-

26982011000100007\&lng=es\&tlng=es.

Hernández, R., Fernández, C. y Baptista, M. (2014). Metodología de la investigación. $6^{\mathrm{a}}$. Ed. McGRAW-HILL. México D.F.

Legorreta, L., Ortega, A., SUÁREZ, R., y García, S. (2019). Terminal Efficiency in Educational Programs of Economic Administrative Sciences of the Technological Institute of Campeche. Journal-Republic of Colombia 2019, 5-9: 8-14.

Martínez-Otero, P. V. (2009). Diversos condicionantes del fracaso escolar en educación secundaria. Revista Iberoamericana de Educación, 51, 67-85.

Salazar F., Paulina, Manterola D., Carlos, Quiroz S., Guissela, García M., Nayely, Otzen H., Tamara, Mora V., Miriann, \& Duque P., Galo. (2019). Estudios de cohortes. $1^{a}$ parte. Descripción, metodología y aplicaciones. Revista de cirugía, 71(5), 482-493 Recuperado en abril 15, $2020 \quad$ de https://dx.doi.org/10.35687/s2452-

45492019005431

Sánchez-Olavarría, César. (2019). Trayectorias escolares en el nivel medio superior: el caso de una institución pública. Revista iberoamericana de educación superior, 10 (28), 66-81. Recuperado en abril 1, 2020 de https://dx.doi.org/10.22201/iisue.20072872e.20 19.28.429

TecNM (2014). Programa Institucional de Innovación y Desarrollo 2013-2018 del Tecnológico Nacional de México. Sfera Creativa S.A. de C.V. México.
TecNM (2015). Manual de Lineamientos Académico-Administrativos del Tecnológico Nacional de México. Planes de estudio para la formación y desarrollo de competencias profesionales. TecNM. México.

TecNM (2018). Programa de Trabajo Anual 2019 del Tecnológico Nacional de México. Recuperado en abril 21, 2020 de https://www.tecnm.mx/pdf/PTA2019-TecNMEFF.pdf

TecNM (2015). Lineamiento para la Evaluación y Acreditación de Asignaturas Versión 1.0, Planes de Estudio 2009-2010. Recuperado en abril 21, 2020 de https://www.culiacan.tecnm.mx/wpcontent/uploads/2016/01/LINEAMIENTO-

PARA-LA-EVALUACION-YACREDITACION-DE-ASIGNATURASAGOSTO-2015.pdf

Tovar, T., Gutiérrez, S., y Sustay, C. (2017). Trayectoria escolar en una cohorte generacional de licenciatura. Memorias del Encuentro Internacional de Educación a Distancia. Año. 5, núm. 5, diciembre 2016noviembre 2017, Recuperado en abril 21, 2020 de

http://www.udgvirtual.udg.mx/remeied/index.p $\mathrm{hp} /$ memorias/article/view/296

Velazco, S. (2016). El rendimiento académico en la etapa de educación secundaria en Chiapas (México): una aproximación comparativa a los resultados internacionales, nacionales y a la cotidianeidad escolar. Tesis doctoral. Universidad Autónoma de Barcelona. Recuperado en abril 22, 2020 de https://ddd.uab.cat/pub/tesis/2016/hdl_10803_3 99337/gsvl1de1.pdf

Zandomeni, N., \& Canale, S. (2010). Divulgación científica: Las trayectorias académicas como objeto de investigación en las instituciones de educación superior. Ciencias Económicas, $\quad 2 \quad$ (13), 59-65. https://doi.org/10.14409/ce.v2i13.1152. 